\title{
Influência de sessões de alongamento sobre os níveis de flexibilidade de idosos sedentários
}

\author{
Bernardo Minelli Rodrigues*, Rodrigo Almeida Aguiar**, Gabriela Rezende de Oliveira Venturini***, \\ Dihogo Gama de Matos ${ }^{* * * *}$, Felipe José Aidar ${ }^{* * * * *}$, Mauro Lúcio Mazini Filho ${ }^{* * * * * *}$
}

\section{Resumo}

O envelhecimento é um fenômeno fisiológico que afeta drasticamente todo o organismo, principalmente as qualidades físicas: força, flexibilidade, indicadores antropométricos e cardiorrespiratórios. O objetivo deste estudo foi analisar a treinabilidade da flexibilidade em idosos submetidos a sessões de alongamentos. Participaram do estudo dez indivíduos $(\mathrm{n}=10)$ do gênero masculino, escolhidos aleatoriamente, com idade entre 62 e 68 anos, que não tivessem participado de programas com exercícios de alongamento. As intervenções ocorreram ao longo de doze semanas, duas vezes por semana, tendo cada sessão de exercícios duração de 30 a 45 minutos. O IPAQ (classificação como sedentários) e liberação médica foram utilizados como critério de inclusão. Foram realizados o pré e o pós-teste por meio da avaliação de goniometria coxofemoral. Foi feita a verificação da homogeneidade da amostra mediante o teste de Shapiro Wilk e o teste $\mathrm{t}$ para amostras pareadas $(p<0,05)$, sendo utilizado o SPSS $®$ for Windows 15.0. Foram registradas diferenças estatísticas significativas, evidenciando um ganho considerável na amplitude articular do quadril após a utilização das sessões de alongamentos na região coxofemoral. No pré-teste, foi encontrada uma média de 39,80 $\pm 7,8$ graus na articulação do quadril. No final das doze semanas de aplicação do alongamento (pós-teste), observou-se média de 55,80 \pm 7,3 graus. Concluiu-se que houve um ganho significativo na amplitude articular dos participantes, evidenciando que a realização de exercícios de alongamento reflete em benefícios à amplitude angular e melhora nos níveis de flexibilidade.

Palavras-chave: Idosos. Envelhecimento. Flexibilidade.

* Docente do curso de Ed. Física das Faculdades Sudamérica, Cataguases - MG e docente da Funita, Itaperuna - RJ. Endereço para correspondência: Rua Edson Barbosa Resende, 49, Esteves, CEP: 36700-000, Leopoldina - MG. E-mail: bernadimmr@yahoo.com.br.

* Discente do curso de Pós-Graduação em Personal Trainer e prescrição de exercícios para grupos especiais pelo Centro Universitário de Volta Redonda - UNIfoa - Unidade IF.

**: Discente do curso de Bacharel em Educação Física pela Faculdade de Minas - Faminas, Murié - MG.

**** Mestre em Educação Física e Desporto, Doutorando em Ciências do Desporto pela Universidade Trás-os-Montes e Alto Douro, UTDA - Portugal.

*****: Doutor em Ciências do Desporto pela Universidade Trás-os-Montes e Alto Douro, Portugal. Docente do curso de Educação Física pela Universidade Presidente Antônio Carlos, Unipac, Uberlândia - MG e docente do curso de Educação Física pelo Centro Universitário do Triângulo, Unitri, Uberlândia - MG. Mestre em Educação Física e Desporto, doutorando em Ciências do Desporto pela Universidade Trás-os-Montes e Alto Douro, UTad, Portugal. Docente do curso de Educação Física das Faculdades Sudamérica, Cataguases - MG, do curso de Educação Física da Presidente Antônio Carlos, Fapac, Leopoldina - MG. Docente das Faculdades Integradas de Cataguases, FIC/UNIS, Cataguases - MG.

$\hookrightarrow$ doi:10.5335/rbceh.2012.022 


\section{Introdução}

O Instituto Brasileiro de Geografia e Estatística (IBGE, 2004) afirma que, entre 1950 e 2025, a população de idosos crescerá 16 vezes, enquanto a população total aumentará somente 5 vezes.

O corpo humano requer uma postura estável e balanceada com suporte para exercer seus movimentos coordenados e suas diversas funções. (CAÇADO, 1999). O envelhecimento é um fenômeno fisiológico que afeta drasticamente todo o organismo, principalmente as qualidades físicas: força, flexibilidade, indicadores antropométricos e cardiorrespiratórios. (PAPALEO NETTO, 2002; MAZINI FILHO et al., 2006).

Mazini Filho et al. (2010) e Zakas et al. (2001) demonstraram que as modificações sofridas no processo do envelhecimento estão diretamente relacionados à diminuição da autonomia e da independência funcional dos indivíduos, o que acaba por comprometer as capacidades físicas dos idosos. Programas de exercícios físicos de alongamento e flexibilidade são contemplados e tendem a permitir que essas pessoas apresentem nível funcional relativamente melhor, oferecendo maior proteção contra diversas patologias e fatores de risco, particularmente os relacionados ao sistema osteomioarticular. (SCHUBACK; HOOPER; SALISBURY, 2004; MATOS et al., 2012). Atividades de força muscular e exercícios aeróbicos também fazem parte de estratégias que visam à independência funcional e à manutenção das capacidades físicas dessa população. (MAZINI FILHO et al., 2010). Esses mesmos autores demonstraram o quão benéfico é para a pessoa idosa a combinação das valências físicas numa mesma sessão de treinamento, conforme aborda o presente experimento.

Os exercícios de alongamento, embora não previnam diretamente a ocorrência de lesões (VAUGHAN; DAVIS; O'CONNOR, 1993; ANDERSEN, 2005), auxiliam no aumento e na manutenção da amplitude de movimentação articular (ALTER, 1999; GRAHAM, 2000; MATOS et al., 2012), podendo diminuir a limitação mecânica causada por alterações nas características viscoelásticas da musculatura em atividades dinâmicas. (VIEL, 2001). Contudo, ainda se percebem algumas lacunas na literatura, pelo fato de haver poucos trabalhos tratando dos alongamentos relacionados aos adultos e idosos na população brasileira. Sendo assim, o objetivo do presente estudo foi analisar alterações na flexibilidade de idosos antes e depois de serem submetidos a sessões de alongamentos durante doze semanas.

\section{Materiais e métodos}

\section{Amostra}

Participaram do estudo dez voluntários $(\mathrm{n}=10)$ do gênero masculino com idade entre 62 e 68 anos $( \pm 2,3$ anos), que não estavam engajados em outros programas de atividades físicas sistematizadas, a não ser caminhadas esporádicas. 
Tabela 1 - Dados antropométricos dos indivíduos.

\begin{tabular}{lccccc}
\hline & $\mathrm{N}$ & Idade (anos) & Peso $(\mathrm{kg})$ & Estatura $(\mathrm{cm})$ & IMC $\left(\mathrm{kg} \cdot \mathrm{m}^{-2}\right)$ \\
\hline Voluntários & 10 & $66,2 \pm 10,8$ & $55 \pm 6$ & $165 \pm 8$ & $18 \pm 5$ \\
\hline
\end{tabular}

Como critério de inclusão no estudo, estabeleceu-se a liberação médica. Além disso, os participantes deveriam ser do gênero masculino e ter idade superior a 60 anos por ocasião do início da pesquisa. Os pesquisados que apresentaram alguma patologia somente foram aceitos no programa estando clinicamente estáveis. Foram incluídos no estudo apenas os sujeitos classificados como insuficientemente ativos ou sedentários. Como critérios de exclusão, utilizou-se: problemas osteomioarticulares que comprometessem a execução das atividades ou que apresentassem potencial risco de agravamento pelo programa de exercícios físicos propostos; intervenção cirúrgica para revascularização; reposição hormonal; uso de qualquer tipo de ergogênico ilícito. O estudo não controlou a dieta dos participantes, nem foi utilizado como critério de exclusão o uso de fármacos de forma continuada ou não, uso este devido a problemas crônicos ou agudos, principalmente em decorrência de problemas em consequência do envelhecimento.

$\mathrm{O}$ presente trabalho atende às Normas para Realização de Pesquisa em Seres Humanos, Resolução 196/96, do Conselho Nacional de Saúde, e foi submetido ao Comitê de Ética em Pesquisa da Universidade Federal de Juiz de Fora, Minas Gerais, Brasil, obtendo aprovação sob o número 0284.0.180.000-08.

\section{Instrumentos}

\section{Flexibilidade}

Foi utilizado o protocolo de goniometria proposto pelo Labifie (DANTAS; CARVALHO; FONSECA, 1997), para verificação da capacidade máxima de amplitude articular, em graus, no movimento de flexão da articulação coxofemoral (quadril) unilateralmente nos voluntários. O goniômetro utilizado para tomada das medidas de flexibilidade foi o Lafayette ${ }^{\circledR}$ (USA), que registra uma amplitude articular de $360^{\circ}$. A flexibilidade foi mensurada de maneira passiva, e a escolha da articulação deu-se pelo fato de o processo deletério do envelhecimento acometer principalmente as fibras de contração rápida, promovendo diminuição da flexibilidade dos membros inferiores, o que acarreta dificuldades na deambulação. (MAZINI FILHO et al., 2006).

\section{Antropometria}

Para as variáveis antropométricas, todos os indivíduos foram medidos e pesados descalços, trajando roupas leves numa balança Filizola Brasil@ com precisão de 100 g. A medida de estatura foi realizada em estadiômetro da marca Sanny® com precisão de $1 \mathrm{~cm}$. O avaliado foi posicionado no estadiômetro com braços estendidos ao longo do corpo, pés 
unidos e centralizados, cabeça, nádegas e calcanhares encostados na parede e com os olhos mantidos no plano horizontal (plano de Frankfört). Com base nessas medidas, o índice de massa corporal (IMC) foi determinado pelo quociente de massa corporal/(estatura), sendo a massa corporal expressa em quilogramas $(\mathrm{kg})$ e a estatura, em metros (m).

\section{Nível de atividade física}

Para a verificação do nível de atividades físicas, foi utilizado o International Physical Activity Questionnaire (IPAQ), na versão curta, contendo perguntas em relação à frequência e à duração das atividades físicas. Os indivíduos foram classificados como muito ativos, ativos, irregularmente ativos e sedentários, com base em respostas a questões fechadas. As perguntas do questionário estão relacionadas às atividades realizadas na semana anterior à sua aplicação. Os participantes tiveram seus dados tabulados, avaliados e foram posteriormente classificados de acordo com a orientação do próprio IPAQ. O questionário visa à classificação dos indivíduos quanto à prática de atividades físicas, sendo dividido desde sedentários até muito ativos, conforme a classificação a seguir:

\section{Classificação do IPAQ}

- sedentário: não realiza qualquer atividade física por pelo menos 10 minutos contínuos durante a semana;

- insuficientemente ativo: consiste em classificar os indivíduos que praticam atividades físicas por pelo menos 10 minutos contínuos por semana, porém de maneira insuficiente para ser classificado como ativos. Para classificar os indivíduos nesse critério, são somadas a duração e a frequência dos diferentes tipos de atividades (caminhadas + moderada + vigorosa). Essa categoria divide-se em dois grupos:

- ativo: cumpre as seguintes recomendações: a) atividade física vigorosa $->3$ dias/semana e $>20$ minutos/sessão; b) moderada ou caminhada $->5$ dias/semana e $>30$ minutos/sessão; c) qualquer atividade somada: $>5$ dias/semana e > $150 \mathrm{~min} /$ semana;

- muito ativo: cumpre as seguintes recomendações: a) vigorosa $->5$ dias/semana e > $30 \mathrm{~min} / \mathrm{sessão}$; b) vigorosa $->3$ dias/semana $\mathrm{e}$ $>20 \mathrm{~min} / \mathrm{sessão} \mathrm{+} \mathrm{moderada} \mathrm{e} \mathrm{ou}$ caminhada 3 a 5 dias/semana e > $30 \mathrm{~min} / \mathrm{sessão.}$

\section{Procedimentos}

Intervenção

Os voluntários foram submetidos a avaliação médica e, após a liberação para a prática da atividade física, foi realizada a avaliação do nível de atividade física por meio do IPAQ, por um único avaliador com experiência mínima de 20 testes, somente tendo sido aceitos no programa os classificados como sedentários.

Após a inclusão no programa, os voluntários realizaram sessões de alongamento por doze semanas, com a frequência de duas vezes semanais e duração de 30 a 45 minutos. Cada sessão envolveu alongamentos passivos na região coxofe- 
moral, contendo 10 segundos por série, para ganho de amplitude articular e alongamento muscular. Foram realizados três exercícios de alongamento, alternando os hemisférios. Contudo, o programa ainda contava com um aquecimento com ativação cardiocirculatória aeróbica (5 min); exercícios de resistência adaptados (10 min); atividades de coordenação (15-20 min); exercícios respiratórios e de relaxamento (5-10 min); e hidratação em dois momentos distintos (25 minutos após o início e ao final da sessão). Vale ressaltar que a prática sistematizada entre os exercícios realizados foi escolhida de acordo com as sugestões de Mazini Filho et al. (2010), que abordaram exercícios físicos combinados para verificação de variáveis físicas e fisiológicas. No presente experimento, optou-se apenas por mensurar a capacidade física flexibilidade. É possível que a soma dos exercícios físicos realizados exerça potenciais resultados finais em nossa variável investigada, podendo essas outras atividades prescritas ser consideradas como limitações de nosso estudo, uma vez que não foram controlados grupos que praticaram apenas atividades de alongamentos na região coxofemoral.

As medidas foram tomadas antes do início do programa e após 12 semanas de intervenção, sempre pelo mesmo pesquisador, respeitando, também, os mesmos horários.

\section{Flexibilidade}

Para o teste de flexibilidade do quadril com o grupo de idosos, a medida foi feita com cada voluntário deitado em decúbito dorsal em uma maca de ree- ducação postural global (RPG). O goniômetro foi colocado em seu eixo central ao nível do trocanter maior do fêmur, com uma haste fixa na parte lateral do tronco sobre o prolongamento da linha axilar, e a outra, na face externa da coxa em sua linha mediana, aplicando-se duas avaliações.

$\mathrm{Na}$ primeira avaliação realizada, estabilizou-se o membro contralateral na tentativa de se evitar possíveis interferências nos resultados da região coxofemoral. Os resultados obtidos da medida de cada voluntário foram registrados em uma ficha de avaliação para posterior reavaliação.

Ao término do período de aplicação das sessões de alongamentos na região coxofemoral, foi realizado um pós-teste na amostra, utilizando o mesmo protocolo (DANTAS; CARVALHO; FONSECA, 1997) do pré-teste para registrar a amplitude articular atual do quadril de todos os voluntários.

Os resultados da reavaliação foram cuidadosamente analisados e comparados com os da avaliação inicial, obtendo-se, assim, conclusões expressivas. Todas as avaliações no pré e pós-teste foram realizadas em laboratório climatizado com temperatura ambiente mantida entre 16 e $20{ }^{\circ} \mathrm{C}$ e obedeceram a uma reprodutividade parecida, sendo realizadas no período da tarde, às $16 \mathrm{~h}$. No dia da avaliação, conforme orientação recebida, nenhum voluntário havia praticado qualquer atividade física, para evitar ganhos ou perdas nos resultados encontrados entre os testes. 


\section{Estatística}

A verificação da homogeneidade da amostra foi feita por meio do teste de Shapiro Wilk. Diante da normalidade, procedeu-se ao teste $t$ para amostras pareadas. Adotou-se como nível de significância $\mathrm{p}<0,05$, utilizando para a análise o programa SPSS® for Windows versão 15.0 .

\section{Resultados}

A análise e a comparação dos dados coletados no pré e pós-teste evidenciaram diferenças estatísticas relevantes, assinalando um ganho considerável na amplitude articular do quadril após a utilização das sessões de alongamentos na região coxofemoral dos idosos mediante a intervenção proposta (Figura 1).

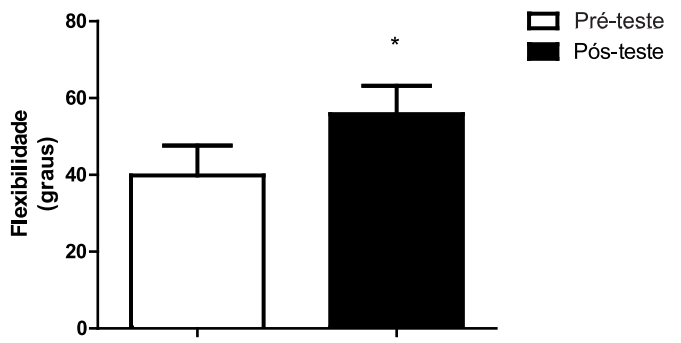

Figura 1 - Avaliação da amplitude articular, em graus, no movimento de flexão da articulação coxofemoral.

* diferença significativa $\mathrm{p}<0,0001$ pré e pós-teste.

No pré-teste, foi encontrada uma média de 39,80 $\pm 7,8$ graus na articulação do quadril. No final das 12 semanas de aplicação do alongamento (pós-teste), observou-se média de $55,80 \pm 7,3$ graus, o que mostrou a eficiência da prescrição das atividades no presente experimento.

\section{Discussão}

No presente estudo, constatou-se que todos os indivíduos apresentaram melhoras significativas na flexibilidade, da ordem de $39,80 \pm 7,8$ graus para 55,80 $\pm 7,3$ graus. O programa de atividade física proposto, associado à utilização das sessões de alongamentos na região coxofemoral, mostrou-se eficiente em ganhos de amplitude de movimento dessa articulação nos idosos participantes.

Segundo Shephard (1998), durante a vida ativa, adultos tendem a perder cerca de 8 a 10 centímetros de flexibilidade na região lombar e no quadril, quando medidos por meio do teste de alcance máximo "sit and reach". Os fatores que contribuiriam para essa perda seriam a maior rigidez de tendões, ligamentos e cápsulas articulares, devido a deficiências no colágeno. $\mathrm{O}$ mesmo autor menciona que a restrição na amplitude do movimento das grandes articulações progride de forma mais enfática com o envelhecimento, e, muitas vezes, a independência funcional acaba por ser diminuída. Assim, uma das estratégias para conservar a flexibilidade consiste na realização de movimentos em toda a amplitude das principais articulações.

Geraldes et al. (2008) investigaram a relação entre a amplitude articular da flexão e extensão das articulações glenoumerais (GU) e coxofemorais (CF) e o desempenho funcional (DF) em 22 idosas funcionalmente independentes e fisicamente ativas. Os autores constataram correlações significantes $(p<0,05)$ entre a flexibilidade ativo-assistida de $\mathrm{CF}$ e alguns testes específicos de DF. 
Nenhuma relação foi identificada para DF e GU.

De acordo com Cristopoliski et al. (2008), o processo do envelhecimento seria caracterizado pela redução gradativa da eficiência do aparelho locomotor, que ocorre pela diminuição na flexibilidade, dentre outros fatores. Ao verificar o efeito de uma sessão de exercícios de alongamentos dos músculos extensores e flexores do quadril sobre a marcha de indivíduos idosos, foram avaliadas cinco idosas $(67,0 \pm 3,8$ anos; $1,59 \pm 0,07 \mathrm{~m}$; $64,3 \pm 15,3 \mathrm{Kg}$ ). A marcha dos sujeitos foi analisada antes e depois dos exercícios de alongamentos. O protocolo consistiu em uma sessão de três séries de exercícios de alongamentos de 30 segundos com o método estático, feitos para os músculos flexores e extensores da articulação do quadril. Após a sessão de flexibilidade, a marcha das participantes apresentou menor pico de inclinação anterior da pelve, maior pico de extensão e amplitude total de movimento da articulação do quadril, maior amplitude de movimento do joelho com maior ângulo de flexão durante a fase de balanço médio e maior altura de separação do pé ao solo (aumento este de $28,6 \%$ ).

Resultado semelhante foi encontrado por Geraldes et al. (2007), que verificaram a relação entre a flexibilidade multiarticular e o desempenho funcional de 30 idosas (68 \pm 1 anos) funcionalmente independentes e fisicamente ativas, sendo constatadas correlações entre a flexibilidade e atividades como amarrar o sapato, bem como entre a flexibilidade e o desempenho funcional. Os autores concluíram que a flexibilidade tende a melhorar com os exercícios de alongamento, interferindo diretamente, ainda, nas atividades da vida dos idosos.

Farinatti e Lopes (2004) verificaram a associação de amplitude e cadência do passo com componentes da aptidão muscular (flexibilidade, força e resistência muscular de membros inferiores), avaliando 25 mulheres de 60 a 86 anos de idade (média $=79 \pm 7$ anos). Os resultados indicaram que a flexibilidade tem relação direta com a amplitude da passada.

Matos et al. (2012), ao estudarem a flexibilidade de mulheres de meia-idade, compararam dois grupos que realizaram treinamento em dois dias semanais e outro que os realizou em três dias semanais. Verificaram que o grupo que mais treinou obteve melhoras superiores ao que menos treinou. Vale ressaltar que o instrumento aplicado pelos autores se diferencia do empregado na presente investigação, pois naquele caso o teste de sentar e alcançar foi o recurso utilizado, enquanto neste optou-se pelo uso do goniômetro. Outra diferença está relacionada ao gênero, pois o trabalho de Matos et al. (2012) teve como foco as mulheres, ao passo que nesta investigação os avaliados são homens. Outra diferença a ser levada em consideração é que os autores realizaram um estudo por um período de 15 meses, enquanto neste experimento adotou-se o período de 12 semanas apenas. Porém, em ambas as investigações, resultados satisfatórios foram encontrados na capacidade física flexibilidade.

A despeito de nossos achados, Ueno et al. (2000) demonstraram que 12 se- 
manas de trabalho de alongamentos não seriam suficientes para o aumento da flexibilidade em idosos. Assim, uma vez que a literatura ainda não é conclusiva no que se refere a tempo de intervenção e modelos específicos de treinamento, mais investigações com o maior controle possível de variáveis devem ser rigorosamente empreendidas, mediante utilização de instrumentos e avaliações de populações de idosos ativos e inativos, para que sejam traçados resultados para essa capacidade física de um modo geral.

\section{Conclusão}

Por meio da intervenção que contemplou exercícios físicos combinados numa mesma sessão de treinamento, foi possível concluir que campos da utilização de alongamentos específicos para a região coxofemoral influenciaram de forma positiva no ganho de amplitude de movimento dessa articulação por parte dos idosos participantes. A realização de outras pesquisas na mesma linha de raciocínio seria altamente enriquecedora para esclarecer algumas lacunas que ainda cercam o fenômeno do envelhecimento.

\section{Influence of stretching sessions on levels of flexibility in sedentary elderly}

\section{Abstract}

Aging is a physiological phenomenon that drastically affects the whole body, especially the physical qualities: strength, flexibility, cardiorespiratory and anthropometric indicators. The aim of this study was to assess the trainability of flexibility in the elderly undergoing stretching sessions. The study included 10 subjects, randomized, (n $=10$ ), aged between 62 and 68 year old male, who had not participated in programs with stretching exercises. Were done 12 weeks of intervention by stretching exercises performed twice a week, lasting between 30 and 45 minutes each session. The IPAQ (classified as sedentary) and medical release was used as criteria for inclusion. Was performed pre and post test by evaluating coxofemural goniometry. Was made to verify the homogeneity of the sample through the Shapiro Wilk, t test for paired samples, $p<0.05$, by using the spss for Windows 15.0. Statistically significant difference was recorded, showing a considerable gain in amplitude of the hip after the use of stretching sessions in the hip region. In the pre-test found an average of $39.80 \pm 7.8$ degrees in the hip joint. At the end of 12 weeks of application of stretch (post-test), there was an average of $55.80 \pm 7.3$ degrees. It was concluded that there was a significant gain in joint range of participants, showing that the achievement of stretching exercises, is beneficial with respect to amplitude and angular improvement in levels of flexibility.

Keywords: Elderly. Aging. Flexibility.

\section{Referências}

ALTER, M. J. Ciência da flexibilidade. 2. ed. Porto Alegre: Artmed, 1999.

ANDERSEN, J. C. Stretching before and after exercise: effect on muscle soreness and injury risk. Journal of Athetic Training, v. 40, n. 3, p. 218-220, 2005.

CAÇADO, F. S. X. Noções práticas de geriatria: uma abordagem na saúde do idoso. Belo Horizonte: Coopme; Coffito, 1999. Disponível em: <http://www.coffito.org.com.br/1999>. Acesso em: 22 mar. 2010.

CRISTOPOLISKI, F. et al. Efeito transiente de exercícios de flexibilidade na articulação do quadril sobre a marcha de idosas. Revis- 
ta Brasileira de Medicina do Esporte, São Paulo, v. 14, n. 2, p. 139-144, 2008.

DANTAS, E. H. M.; CARVALHO, J. L. T.; FONSECA, R. M. O protocolo de Labifie de goniometria. Revista Treinamento Desportivo, Curitiba, v. 3, n. 4, p. 34, 1997.

FARINATTI, P. T. V.; LOPES, L. N. C. Amplitude e cadência do passo e componentes da aptidão muscular em idosos: um estudo correlacional multivariado. Revista Brasileira de Medicina do Esporte, São Paulo, v. 10, n. 5, p. 389-400, 2004.

GERALDES, A. A. R. et al. Correlação entre a flexibilidade multiarticular e o desempenho funcional de idosas fisicamente ativas em tarefas motoras selecionadas. Revista Brasileira de Cineantropometria \& Desempenho Humano, Florianópolis, v. 9, n. 3, p. 238-243, 2007.

GERALDES, A. A. R. et al. Correlação entre flexibilidade das articulações glenoumerais e coxofemorais e o desempenho funcional de idosas fisicamente ativas. Revista Brasileira de Fisioterapia, São Carlos, v. 12, n. 4, p. 274-282, 2008.

GRAHAM, B. J. A randomize e trial of pre exercise stretching for prevention of lower limb injury. Medicine Science and Sports Exercise, v. 32, n. 2, p. 271-277, 2000.

INSTITUTO BRASILEIRO DE GEOGRAFIA E ESTATÍSTICA (IBGE). Diretoria de Pesquisa. Coordenação de População e Indicadores Sociais. Projeção de população do Brasil por sexo e idade para o período de 1980-2050. Rio de Janeiro: IBGE, 2004.

MATOS, D. G. et al. Efeito de diferentes frequências semanais de treinamento físico geral sobre a flexibilidade de mulheres de meia-idade. Revista Brasileira de Cineantropometria e Desempenho Humano, v. 14, n. 5, p. 582-591, 2012.

MATSUDO, S. M. et al. Questionário Internacional de Atividade Física (IPAQ): estudo de validade e reprodutibilidade no Brasil. Revista Brasileira de Atividade e Saúde, Florianópolis, v. 6, n. 2, p. 5-18, 2001.
MAZINI FILHO, M. L. et al. Os benefícios do treinamento de força na autonomia funcional do indivíduo idoso. Revista de Educação Fisica, Rio de Janeiro, v. 134, [s. n.], p. 57-68, 2006.

MAZINI FILHO, M. L. et al. Efeito de atividades físicas combinadas na autonomia funcional, índice de massa corporal e pressão arterial de mulheres idosas. Geriatria e Gerontologia, Rio de Janeiro, v. 4, n. 2, p. 69-75, 2010.

PAPALEO NETTO, M. Gerontologia: a velhice e o envelhecimento em uma visão globalizada. São Paulo: Atheneu, 2002.

SCHUBACK, B.; HOOPER, J.; SALISBURY, L. A caparison of self stretch incorporating proprioceptive neuromuscular facilitation components and a therapist applied PNF technique on hamstring flexibility. Physiotherapy, v. 90, n. 3, p. 151-157, 2004.

SHEPHARD, R. J. Aging and exercise: encyclopedia of sports, medicine and science. Internet Society for Sport Science, 1998. Disponível em: <http://sportsci.org>. Acesso em: 20 jan. 2009.

UENO, L. M. et al. Análise dos efeitos quantitativos e qualitativos de um programa de educação física sobre a flexibilidade do quadril em indivíduos com mais de 60 anos. Motriz, Rio Claro, SP, v. 6, n. 1, p. 9-16, 2000.

VAUGHAN, C. L.; DAVIS, B. L.; O’CONNOR, J. C. Dynamics of human gait. Illinois, Champaign: Human Kinetics, 1993.

VIEL, E. A marcha humana, a corrida e o salto: biomecânica, investigações, normas e disfunções. Barueri, SP: Manole, 2001.

ZAKAS, A. et al. Effects of stretching exercise during strength training in pre pubertal, pubertal and adolescent boys. Journal of Bodywork and Movement Therapies, v. 6, n. 3, p. 170-176, 2001. 